\title{
Atividades para o ensino básico envolvendo Relações de Girard e Aritmética
}

\author{
Activities for the basic education involving Relations of Girard and Arithmetic
}

\author{
Rosipléia Souza Santos ${ }^{1}$ e Kisnney Emiliano de Almeida ${ }^{2}$ \\ 1,2 Universidade Estadual de Feira de Santana. BA, Brasil \\ rosipleia@hotmail.com
}

\begin{abstract}
Resumo
Este trabalho tem por objetivo construir uma proposta didática de como ensinar métodos de encontrar as raízes inteiras ou racionais de polinômios de grau superior a dois utilizando propriedades de números inteiros aprendidas no estudo de aritmética $e$ as relações entre coeficientes e raízes de polinômios conhecidas como Relações de Girard, com mínima aplicação de fórmulas prontas. Dessa maneira, é dada ao aluno a oportunidade de exercitar simultaneamente e de maneira dinâmica os conceitos básicos de álgebra e aritmética presentes no ensino básico.
\end{abstract}

Palavras-chave: Ensino de Matemática, Polinômios, Relações de Girard, Aritmética.

\begin{abstract}
The objective of this work is to build a didatic proposal for teaching methods to fing the integer or racional roots of polynomials of degree greater than two by using properties of integers learned on the studies of Arithmetic and the relations between coeficients and roots of polynomials known as Relations of Girard, with minimal application of formulas. Thus the student is given the opportunity to exercise simultaneously and dynamically the basics of algebra and arithmetic present in basic education.
\end{abstract}

Keywords: Mathematics Teaching, Polynomials, Relations of Girard, Arithmetic 


\section{Introdução}

O ensino da álgebra na educação básica tem sido um desafio para nós, professores da rede pública. Nossos alunos demonstram uma certa recusa por acharem que matemática está diretamente ligada a números e que as letras apenas atrapalham. Em se tratando de polinômios, conteúdo estudado inicialmente no oitavo ano do ensino fundamental e aprofundado no terceiro ano do ensino médio, uma das maiores dificuldades é encontrar as raízes quando este polinômio tem grau superior a dois, visto que as fórmulas para encontrar tais raízes não fazem parte do conteúdo programático. Na seção 2, faremos um breve histórico a respeito de matemáticos como Bhaskara, Tartaglia, Cardano e Ferrari, que contribuíram muito para o avanço do estudos dos polinômios descobrindo os métodos que hoje nos dão fórmulas que, embora bastante trabalhosas, podem ser usadas para obter as raízes de equações do terceiro e quarto graus. $\mathrm{Na}$ seção 3, faremos brevemente a exposição das demonstrações das fórmulas mencionadas anteriormente. Na seção 4, faremos uma exposição das relações entre as raízes e os coeficientes dos polinômios, chamadas relações de Girard, que são a principal ferramenta na resolução das atividades que serão propostas na seção seguinte. Como um dos nossos objetivos é desenvolver o raciocínio lógico do educando levando-o a encontrar estratégias para resolução de problemas por caminhos que não sejam apenas aplicação de fórmulas prontas, destinamos a seção 5 para isto. Temos algumas propostas pedagógicas de atividades com as devidas resoluções, que podem ser facilmente adaptadas de modo a servirem como modelo para construção de muitas outras atividades. São estratégias de encontrar raízes de polinômios de grau três, quatro, e até seis usando apenas as relações de Girard e conhecimentos aritméticos que os educandos devem trazer de sua vida acadêmica. Temos também algumas atividades onde deve-se encontrar o polinômio, dadas algumas informações acerca do mesmo, entre outras.

\section{Breve Histórico}

Dentre os matemáticos hindus que se destacaram com contribuições do desenvolvimento da álgebra, o que mais facilmente nos lembramos é Bhaskara, por estar ligado à fórmula geral da solução das equações polinomiais do segundo grau. No entanto, a fórmula que leva seu nome não foi descoberta por ele, conforme o próprio relatou no século XII. Um século antes, o matemático hindu Sridhara (c. 870, Índia - c. 930 Índia) teria encontrado a mencionada fórmula e publicado em uma obra que se perdeu.

A ideia de encontrar uma forma de reduzir uma equação polinomial de $2^{\circ}$ grau para o primeiro através da extração de raízes quadradas foi o instrumento usado com sucesso pelos hindus na busca pela fórmula geral que conhecemos hoje. Segundo Eves (2002), em textos babilônicos, escritos há cerca de 4000 anos, encontram-se descrições de procedimentos para resolução de problemas envolvendo equações do segundo grau. $\mathrm{O}$ autor menciona também que na Grécia, utilizava-se geometria para resolver tais equações. A partir do início do século IX, matemáticos árabes já haviam se empenhado na resolução de equações do segundo grau, cujos procedimentos utilizaram álgebra e geometria dos gregos, e, em decorrência, fórmulas específicas para tipos diferentes de equação surgiram. Contudo, o aparecimento de um método geral para se obter as raízes de uma equação do segundo grau está situado por volta do final do século XVI.

Eis, então, a fórmula de Bhaskara, que, embora não tenha sido deduzida por ele, imortalizou seu nome.

$$
x=\frac{-b \pm \sqrt{b^{2}-4 a c}}{2 a} .
$$

Segundo Garbi (1997), vencidas as equações do segundo grau, a inesgotável curiosidade dos matemáticos levou-os a conjecturar sobre as formas de resolver as do terceiro. Apesar de não terem encontrado a solução para o problema, os árabes tiveram um importante papel no estudo dessas equações. Girolamo Cardano, nascido em Pavia em 1501 e falecido em Roma em 1576, e Nicoló Fontana, apelidado Tartaglia, nascido em Bréscia em 1500, disputaram pelas equações do terceiro grau.

Por volta de 1510, Scipione Del Ferro, matemático italiano, encontrou uma forma geral de resolver equações polinomiais de terceiro grau do tipo $x^{3}+p x+q=0$, mas não a publicou até sua morte. Seu aluno, Antonio Maria Fior, para o qual Del Ferro revelou a solução antes de morrer, aproveitando-se desse conhecimento desafiou Tartaglia visando ganhar notoriedade, já que este era bastante conhecido por seu talento nos estudos. Sabendo da intenção de Fior, Tartaglia dedicou-se entusiasticamente em encontrar uma solução para as equações das quais seria submetido ao desafio, e foi mais longe, encontrando também um método geral para a resolução das equações do tipo $x^{3}+p x^{2}+q=0$.

O resultado do desafio não poderia ter sido diferente: Tartaglia venceu resolvendo corretamente todos os problemas propostos, enquanto Fior saiu humilhado por não conseguir resolver nenhum dos que lhe foi proposto por se tratar de equações do tipo $x^{3}+p x^{2}+q=0$, sobre as quais não detinha conhecimento algum.

Cardano, que estava escrevendo a Prática Arithmeticae Generalis nesta época, acreditando na impossibilidade de uma solução geral para as equações do terceiro grau, ficou sabendo do ocorrido e resolveu pedir a Tartaglia que revelasse a solução encontrada a fim de publicar em sua obra. Tartaglia não concordou e diante da recusa 
Cardano o insultou gravemente. Algum tempo depois, Cardano investiu em formas de conseguir convencer Tartaglia a revelar as tão cobiçadas fórmulas, até que conseguiu sob juramento de jamais revelá-las, contudo, não foi exatamente o que aconteceu. Quebrando suas promessas e juramentos, Cardano, em 1545, publicou o método revelado por Tartaglia que, nada contente por ter sua obra revelada, publicou sua versão dos fatos e denunciou Cardano por haver traído um sagrado juramento sobre a Bíblia.

Assim como ocorreu com a fórmula de Bhaskara, a fórmula da resolução das equações polinomiais de terceiro grau não recebeu o nome do seu verdadeiro criador, e hoje é conhecida como Fórmula de Cardano.

É importante frisar que os métodos e notações utilizados pelos matemáticos da época eram muito diferentes dos atuais. As soluções de Cardano não foram de fato apresentadas como fórmulas, visto que a notação não envolvia a utilização de símbolos para representar incógnitas e operações: esses elementos eram todos descritos de forma textual. Além disso, as justificativas eram feitas principalmente através de argumentações geométricas e não demonstrações algébricas. Mais ainda, como os conjuntos numéricos como conhecemos hoje ainda não tinham sido plenamente desenvolvidos, as resoluções se dividiam em casos, analisando vários tipos de equações separadamente. Para mais detalhes sobre o tema, conferir (Roque, 2012).

Submetido ao desafio de resolver uma equação de quarto grau, Cardano, após inúmeras tentativas sem sucesso, passou a questão para seu jovem e prodigioso discípulo Ludovico Ferrari. Nascido em 1522 na cidade de Bolonha, Ferrari teve sua brilhante inteligência reconhecida muito cedo por seu mestre e, com sua genialidade, encontrou o método geral para a solução das citadas equações, o qual foi publicado por seu mestre, Cardano, na Ars Magna. Olhando a equação

$$
x^{4}+p x^{2}+q x+r=0
$$

Ferrari, segundo Garbi (1997), procurou reagrupar os termos de modo que nos dois lados da igualdade houvesse polinômios quadrados perfeitos. Se tal reagrupamento fosse possível, seriam extraídas as raízes quadradas, cair-se-ia em equações do $2^{\circ}$ grau e o problema estaria resolvido.

Embora muito trabalhoso, o método desenvolvido por Ferrari nos fornece as soluções de uma equação polinomial de quarto grau apenas com operações algébricas, fato que merece destaque.

Para o caso de equações de grau 5 ou mais, Niels Henrik Abel (Nedstrand,1802 - Froland, 1829) provou que não existem soluções por radicais para encontrar suas raízes, como nos casos anteriores. Mesmo para equações de grau pequeno, os métodos acima podem ser muito trabalhosos e pouco interessantes para o aprendizado do aluno do ensino básico. Ao restringirmos o problema para encontrar raízes inteiras, o mesmo se torna bem mais interessante, por permitir a utilização e exercício de conceitos básicos de aritmética e das Relações de Girard, que serão brevemente apresentadas nas próximas seções.

\section{Soluções gerais de equações poli- nomiais de grau 3 e 4}

Nessa seção, faremos as demonstrações das fórmulas algébricas das soluções das equações de terceiro e quarto graus. Novamente enfatizamos que nem as apresentações nem as justificativas dessas fórmulas foram feitas dessa maneira pelos seus célebres autores: as mesmas foram adaptadas aos dias de hoje. Tais fórmulas e demonstrações podem ser encontradas em vários livros, como por exemplo (Hefez; Villela , 2012).

\subsection{Equação polinomial de terceiro grau}

Para resolver a equação

$$
y^{3}+a_{2} y^{2}+a_{1} y+a_{0}=0,
$$

precisamos primeiro fazer a seguinte mudança de variável:

$$
x=y+\frac{a_{2}}{3}
$$

obtendo a equação equivalente

$$
y^{3}+a_{2} y^{2}+a_{1} y+a_{0}=x^{3}+p x+q,
$$

onde

$$
p=a_{1}-\frac{a_{2}^{2}}{3} \quad \text { e } \quad q=\frac{2 a_{2}^{3}}{27}-\frac{a_{1} a_{2}}{3}+a_{0} .
$$

Dessa forma, basta resolver a equação

$$
x^{3}+p x+q=0 \text {. }
$$

Considerando a solução procurada como soma de duas parcelas $x=A+B$, eleve ambos os membros ao cubo. Assim, obtemos

$$
x^{3}=(A+B)^{3}=A^{3}+B^{3}+3 A B(A+B) .
$$

Substituindo $x=A+B$, obtemos

$$
x^{3}=A^{3}+B^{3}+3 A B x,
$$

donde

$$
x^{3}-3 A B x-\left(A^{3}+B^{3}\right)=0 .
$$

Assim, temos que $p=-3 A B$ e $q=-\left(A^{3}+B^{3}\right.$, de forma que

$$
A^{3} B^{3}=-\frac{p^{3}}{27} \quad \text { e } \quad A^{3}+B^{3}=-q .
$$


Conhecendo a soma e o produto de $A^{3}$ e $B^{3}$, recaindo no clássico problema que se resolve com equações de segundo grau. Dessa forma,

$$
\begin{aligned}
& A^{3}=-\frac{q}{2} \pm \sqrt{\left(\frac{q}{2}\right)^{2}+\left(\frac{p}{3}\right)^{3}} \\
& B^{3}=-\frac{q}{2} \mp \sqrt{\left(\frac{q}{2}\right)^{2}+\left(\frac{p}{3}\right)^{3}} .
\end{aligned}
$$

Como $x=A+B$, obtemos

$$
\begin{array}{r}
x=\sqrt[3]{-\frac{q}{2}+\sqrt{\left(\frac{q}{2}\right)^{2}+\left(\frac{p}{3}\right)^{3}}}+ \\
\sqrt[3]{-\frac{q}{2}+\sqrt{\left(\frac{q}{2}\right)^{2}-\left(\frac{p}{3}\right)^{3}}}
\end{array}
$$

a chamada fórmula de Cardano. Note que as raízes quadradas utilizadas acima podem não assumir valores reais. Para uma análise mais cuidadosa desses casos, conferir (Hefez; Villela, 2012).

\subsection{Equação polinomial de quarto grau}

Nosso objetivo nessa seção é resolver a equação

$$
x^{4}+a_{3} x^{3}+a_{2} x^{2}+a_{1} x+a_{0}=0,
$$

seguindo o método de Ferrari. A estratégia é completar quadrados nos dois membros, reduzindo o problema assim à solução de equações de segundo grau. A equação acima é equivalente a

$$
x^{4}+a_{3} x^{3}=-\left(a_{2} x^{2}+a_{1} x+a_{0}\right) .
$$

Completando o quadrado no primeiro membro e reorganizando o segundo membro, temos

$$
\left(x^{2}+\frac{1}{2} a_{3} x\right)^{2}=\left(\frac{1}{4} a_{3}^{2}-a_{2}\right) x^{2}-a_{1} x-a_{0} .
$$

Somando a expressão $y^{2}+2 y\left(x^{2}+\frac{1}{2} a_{3} x\right)$ a ambos os membros, obtemos

$$
\begin{aligned}
& {\left[\left(x^{2}+\frac{1}{2} a_{3} x\right)+y\right]^{2}=} \\
& \quad\left(2 y+\frac{1}{4} a_{3}^{2}-a_{2}\right) x^{2}+\left(y a_{3}-a_{1}\right) x+\left(y^{2}-a_{0}\right) .
\end{aligned}
$$

Para que o segundo membro seja um quadrado perfeito, assim como o primeiro, precisamos que

$$
\left(y a_{3}-a_{1}\right)^{2}-4\left(2 y+\frac{1}{4} a_{3}^{2}-a_{2}\right)\left(y^{2}-a_{0}\right)=0 .
$$

Escolhendo y como uma das soluções da equação (de terceiro grau) acima, obtemos que a equação (1) é equivalente, para algum $\alpha$ e algum $\beta$ (que dependem da escolha de $y$ ), à equação

$$
\left[\left(x^{2} 2+\frac{1}{2} a_{3} x\right)+y\right]^{2}=(\alpha x+\beta)^{2},
$$

cujas soluções são exatamente as soluções das equações (de segundo grau)

$$
\left(x^{2}+\frac{1}{2} a_{3} x\right)+y=\alpha x+\beta
$$

e

$$
\left(x^{2}+\frac{1}{2} a_{3} x+y=\alpha x-\beta\right),
$$

que podem ser resolvidas usando a fórmula de Bhaskara.

\section{Relações de Girard}

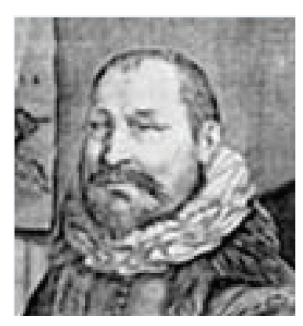

Figura 1: Albert Girard (1595-1632)

Albert Girard (1595-1632), matemático francês, apresentou um importante teorema que relaciona as raízes com os coeficientes de um polinômio.

Para maiores detalhes acerca da álgebra dos polinômios, consultar Domingues (1982), Gonçalves(2008), Hefez (2012), Neto (2012).

Consideremos inicialmente o polinômio do $2^{\circ}$ grau $p(x)=a x^{2}+b x+c$, com $a, b, c \in \mathbb{R}$ e $a \neq 0$, cujas raízes são $x_{1}$ e $x_{2}$. Temos que:

$$
\begin{gathered}
a x^{2}+b x+c=a\left(x-x_{1}\right)\left(x-x_{2}\right) \\
x^{2}+\frac{b}{a} x+\frac{c}{a}=\left(x-x_{1}\right)\left(x-x_{2}\right) \\
x^{2}+\frac{b}{a} x+\frac{c}{a}=x^{2}-\left(x_{1}+x_{2}\right) x+x_{1} x_{2}
\end{gathered}
$$

Igualando os coeficientes, obtemos:

$$
\begin{gathered}
x_{1}+x_{2}=-\frac{b}{a} \\
x_{1} x_{2}=\frac{c}{a},
\end{gathered}
$$

que são equações relacionando diretamente as raízes do polinômio com seus coeficientes. 
Consideremos, agora, o polinômio de grau $3 p(x)=$ $a x^{3}+b x^{2}+c x+d$, com $a, b, c, d \in \mathbb{R}$ e $a \neq 0$, cujas raízes são $x_{1}, x_{2}$ e $x_{3}$. Novamente, temos:

$$
\begin{array}{r}
a x^{3}+b x^{2}+c x+d=a\left(x-x_{1}\right)\left(x-x_{2}\right)\left(x-x_{3}\right) \\
x^{3}+\frac{b}{a} x_{2}+\frac{c}{a} x+\frac{d}{a}=\left(x-x_{1}\right)\left(x-x_{2}\right)\left(x-x_{3}\right) \\
x^{3}+\frac{b}{a} x_{2}+\frac{c}{a} x+\frac{d}{a}=x^{3}-\left(x_{1}+x_{2}+x_{3}\right) x^{2}+ \\
\left(x_{1} x_{2}+x_{1} x_{3}+x_{2} x_{3}\right) x-x_{1} x_{2} x_{3}
\end{array}
$$

Igualando os coeficientes, temos:

$$
\begin{gathered}
x_{1}+x_{2}+x_{3}=-\frac{b}{a} \\
x_{1} x_{2}+x_{1} x_{3}+x_{2} x_{3}=\frac{c}{a} \\
x_{1} x_{2} x_{3}=-\frac{d}{a},
\end{gathered}
$$

que são relações entre os coeficientes e raízes de um polinômio de grau 3.

Generalizando a idéia acima, podemos considerar um polinômio $p(x)=a_{n} x^{n}+a_{n-1} x^{n-1}+\ldots+a_{0}$, sendo $a_{1}, \ldots, a_{n} \in \mathbb{R}, a_{n} \neq 0$ e $n \geq 1$. Podemos então escrever

$$
p(x)=a_{n}\left(x-x_{1}\right)\left(x-x_{2}\right) \cdots\left(x-x_{n}\right) .
$$

Aplicando a propriedade distributiva, reduzindo os termos semelhantes e ordenando o polinômio, temos:

$$
\begin{aligned}
& p(x)=a_{n} x^{n}-x^{n-1}\left(x_{1}+x_{2}+\ldots+x_{n}\right)+ \\
& +x^{n-2}\left(x_{1} x_{2}+x_{1} x_{3}+\ldots+x_{1} x_{n}+x_{2} x_{3}+\ldots+x_{n-1} x_{n}\right)- \\
& -x^{n-3}\left(x_{1} x_{2} x_{3}+x_{1} x_{2} x_{4}+x_{1} x_{3} x_{4}+x_{2} x_{3} x_{4}+\ldots\right. \\
& \left.\quad+x_{n-2} x_{n-1} x_{n}\right)+\ldots+(-1)^{n}\left(x_{1} x_{2} \ldots x_{n-1} x_{n}\right) .
\end{aligned}
$$

Dividindo a expressão por $a_{n}$ e igualando os coeficientes deste último polinômio com o polinômio considerado inicialmente, chegaremos às seguintes relações, conhecidas como relações de Girard:

$$
\begin{gathered}
x_{1}+x_{2}+\ldots+x_{n-1}+x_{n}=-\frac{a_{n-1}}{a_{n}} \\
x_{1} x_{2}+x_{1} x_{3}+\ldots+x_{1} x_{n}+x_{2} x_{3}+\ldots+x_{n-1} x_{n}=\frac{a_{n-2}}{a_{n}} \\
x_{1} x_{2} x_{3}+x_{1} x_{2} x_{4}+x_{1} x_{3} x_{4}+x_{2} x_{3} x_{4}+\ldots \\
+x_{n-2} x_{n-1} x_{n}=-\frac{a_{n-3}}{a_{n}} \\
\vdots \\
x_{1} x_{2} \ldots x_{n-1} x_{n}=(-1)^{n} \frac{a_{0}}{a_{n}}
\end{gathered}
$$

xima seção.

\section{Atividades}

Neste capítulo mostraremos algumas propostas didáticas que vão muito além da aplicação de fórmulas. Aqui pretende-se incentivar o aluno a utilizar conhecimentos aritméticos juntamente com relações de Girard na resolução de problemas e desafios. Para uma melhor fundamentação teórica na parte aritmética, consultar Domingues(1982), Martinez (2013) e Santos (1998).

Seguiremos, nas atividades propostas, abordagens feitas por Dante (2011), Hefez (2012) e Santos (2014).Destinadas a alunos do Ensino Médio, podem ser trabalhadas de forma individual ou em grupos. Comentamos cada questão e explicitamos os conteúdos que podem ser explorados em cada uma delas para que o leitor identifique qual atividade pode ser aplicada em cada série.

Vejamos alguns exemplos onde abordaremos apenas a relação da soma das raízes, divisão de polinômios, potência e valor numérico, ideais para aplicação no início do primeiro ano do ensino médio. A esta altura o professor já deve ter comentado o teorema fundamental da álgebra e deve ter o cuidado de utilizar apenas polinômios com raízes reais. No nosso caso trabalharemos apenas raízes inteiras.

1. Escrever a forma fatorada do polinômio $P(x)=$ $x^{3}-4 x^{2}-4 x+16$, sabendo que duas raízes são simétricas.

Neste polinômio, embora seja possível aplicar a fatoração por agrupamento, utilizaremos a relação da soma das raízes para encontrar a solução.

$$
x_{1}+x_{2}+x_{3}=-(-4)
$$

Como $x_{1}$ e $x_{2}$ são simétricas, temos que $x_{1}+x_{2}=$ 0 , daí temos que $x_{3}=4$.

Se 4 é raiz, podemos dividir $P(x)$ por $x-4$, assim:

$$
x^{3}-4 x^{2}-4 x+16=\left(x^{2}-4\right)(x-4)
$$

Como $x^{2}-4$ é uma diferença de quadrados, sua forma fatorada é $(x-2)(x+2)$, logo, a forma fatorada é

$$
P(x)=(x-4)(x-2)(x+2) .
$$

2. Certo polinômio de coeficiente líder -2 e grau 3 , tem raízes $-1,3$ e 4 . Outro polinômio de coeficiente líder 1 e grau 3 , tem raízes 0 e 3, sendo o 3 uma raiz dupla. Encontre os dois polinômios e determine o mmc entre eles.

Sejam os polinômios $p(x)$ e $g(x)$, então:

$$
p(x)=-2(x+1)(x-3)(x-4)=
$$




$$
\begin{array}{r}
-2 x^{3}+12 x^{2}-10 x-24, \\
g(x)=x(x-3)^{2}=x^{3}-6 x^{2}+9 x .
\end{array}
$$

Para encontrar o $\operatorname{mmc}(p(x), g(x))$, vamos analisar sua forma fatorada e obter:

$$
\begin{gathered}
\operatorname{mmc}(p(x), g(x))=-2 x(x+1)(x-3)^{2}(x-4) \\
=-2 x^{5}+18 x^{4}-46 x^{3}+6 x^{2}+72 x .
\end{gathered}
$$

3. Escreva a forma fatorada do polinômio $P(x)=$ $x^{3}-3 x^{2}-6 x+8$ sabendo que a soma de duas de suas raízes é igual a 5 .

Suponhamos $x_{1}+x_{2}=5$ e substituindo na relação $x_{1}+x_{2}+x_{3}=3$, temos:

$$
5+x_{3}=3 \Longrightarrow x_{3}=-2
$$

Como -2 é raiz, podemos dividir $P(x)$ por $(x+2)$, daí segue que

$$
x^{3}-3 x^{2}-4 x+12=(x+2)\left(x^{2}-5 x+4\right)
$$

Alguns livros trazem a fatoração do trinômio de $2^{\circ}$ grau, além do trinômio quadrado perfeito, onde deve-se procurar as raízes através da soma e do produto das mesmas. Deste modo, para o polinômio $q(x)=x^{2}-5 x+4$ devemos ter duas raízes cuja soma seja 5 e o produto 4 . Assim, as raízes do polinômio são $\{-2,1,4\}$ e sua forma fatorada é $P(x)=(x+2)(x-1)(x-4)$.

4. A professora de matemática colocou uma caixa de bombons num baú e trancou com um cadeado cujo segredo é formado por 3 algarismos. Ela disse que o aluno que acertasse primeiro o segredo ficaria com os bombons e lançou o seguinte desafio: Os algarismos estão em ordem crescente e são as raízes do polinômio $P(x)=$ $x^{3}-11 x^{2}+31 x-21$ e a soma de dois deles é 10.

Podemos escrever $x_{1}+x_{2}=10$ e $x_{1}+x_{2}+x_{3}=$ $-(-11)$, então

$$
10+x_{3}=11 \Longrightarrow x_{3}=1
$$

Efetuando a divisão de $P(x)$ por $x-1$, encontramos:

$$
x^{3}-11 x^{2}+31 x-21=(x-1)\left(x^{2}-10 x+21\right)
$$

Analisando a fatoração do polinômio $q(x)=x^{2}-$ $10 x+21$, temos a soma igual a 10 e o produto igual a 21, logo as raízes procuradas são 3 e 7 . Assim, o segredo do cadeado é 137.

5. Questionada por um aluno a respeito do número de sua casa, a professora de matemática lançou o enigma: $O$ número da minha casa é formado por quatro algarismos que são as raízes do polinômio

$$
P(x)=x^{4}-13 x^{3}+57 x^{2}-99 x+54 .
$$

Os dois primeiros algarismos são iguais, enquanto os dois últimos formam um quadrado perfeito e sua soma é 7 .

Temos que $x_{1}=x_{2}$ e $x_{3}+x_{4}=7$, então:

$x_{1}+x_{2}+x_{3}+x_{4}=13 \Longrightarrow 2 x_{1}+7=13 \Longrightarrow x_{1}=3$

Logo, 3 é a raiz dupla, ou seja $(x-3)^{2}$ divide $P(x)$. Desenvolvendo o produto notável $(x-3)^{2}=$ $x^{2}-6 x+9$ e efetuando a divisão, temos:

$x^{4}-13 x^{3}+57 x^{2}-99 x+54=\left(x^{2}-6 x+9\right)\left(x^{2}-7 x+6\right)$.

Os quadrados perfeitos formados por dois algarismos cuja soma dos algarismos é 7 são 16 e 25 . Substituindo 1 no polinômio:

$$
P(1)=1-13+57-99+54=0
$$

Substituindo 2 no polinômio:

$$
P(2)=16-104+228-198+54=-4
$$

Logo, 1 é raiz do polinômio e o quadrado perfeito procurado é 16. Daí temos que o número da casa da professora é 3316 .

Poderíamos também analisar o quociente da divisão $q(x)=x^{2}-7 x+6$. De acordo com a regra de fatoração do trinômio do $2^{\circ}$ grau, devemos ter duas raízes cuja soma seja 7 e o produto 6 e chegamos aos números 1 e 6 .

Outra solução: Analisando a decomposição em fatores primos do número 54 , temos seus divisores naturais $\{1,2,3,6,9,18,27,54\}$, (já que o número da casa da professora não deve ser um número negativo). Como a soma dos quatro algarismos deve ser 13, os números 18, 27 e 54 são descartados.

Pelas informações passadas no problema, temos uma raiz dupla, que só pode ser 1, 2 ou 3 devido à soma, e as outras duas formam um quadrado perfeito, então:

Caso a raiz dupla seja 1, o único quadrado perfeito que formamos com os divisores restantes é o 36 cuja soma é 9 . Não serve.

Caso a raiz dupla seja 2, podemos formar dois quadrados perfeitos com os divisores restantes: 16, cuja soma é 7 mas a soma das quatro raízes seria $2+2+1+6=11$; ou 36 que já vimos que não pode ser. 
Caso a raiz dupla seja 3, podemos formar o quadrado perfeito 16 cuja soma é 7 e nos fornece a soma das raízes $3+3+1+6=13$

Assim, temos que o número da casa da professora é 3316.

6. Determine o valor de $n \in \mathbb{Z}^{*}$ de modo que o polinômio

$$
p(x)=x^{6}+n x^{4}+2 x^{3}+2 n x^{2}+n x+1
$$

seja quadrado de um polinômio mônico $f(x) \in$ $\mathbb{Z}[x]$, sabendo que os coeficientes de $p(x)$ pertencem a $\mathbb{Z}_{+}$.

Como $\operatorname{gr}(p(x))=6$, ele será o quadrado de um polinômio mônico de grau 3. Seja $f(x)=x^{3}+$ $a x^{2}+b x+c$, então

$$
\begin{array}{r}
f(x)^{2}=x^{6}+a^{2} x^{4}+b^{2} x^{2}+c^{2}+2 a x^{5}+2 b x^{4}+ \\
+2 c x^{3}+2 a b x^{3}+2 a c x^{2}+2 b c x
\end{array}
$$

Agrupando os termos semelhantes, temos:

$$
\begin{aligned}
f(x)^{2}=x^{6}+2 a x^{5} & +\left(a^{2}+2 b\right) x^{4}+(2 c+2 a b) x^{3}+ \\
& +\left(b^{2}+2 a c\right) x^{2}+2 b c x+c^{2} .
\end{aligned}
$$

Comparando $p(x)$ com o quadrado de $f(x)$, temos

$$
\begin{gathered}
2 a=0 \Rightarrow a=0 \\
c^{2}=1 \Rightarrow c= \pm 1
\end{gathered}
$$

mas $-1 \notin \mathbb{Z}_{+}, \log c=1$,

$$
\begin{gathered}
a^{2}+2 b=n \Rightarrow 2 b=n \\
b^{2}+2 a c=2 n \Rightarrow b^{2}=2 n \Rightarrow b^{2}=2 \cdot 2 b .
\end{gathered}
$$

Ou seja, $b \in\{0,4\}$. Para $b=0$, temos $n=0$, que não serve pois $n \in \mathbb{Z}^{*}$. Para $b=4$ temos $n=8$, assim

$$
\begin{array}{r}
p(x)=x^{6}+8 x^{4}+2 x^{3}+16 x^{2}+8 x+1= \\
\left(x^{3}+4 x+1\right)^{2} .
\end{array}
$$

7. Obtenha $a \in \mathbb{Z}$ de modo que $p(x)=x^{4}+a x^{3}+$ $7 x^{2}-a x+1$ seja o quadrado de um polinômio mônico de coeficientes inteiros.

Como gr $(p(x))=4, p(x)$ será o quadrado de um polinômio mônico de grau 2. Seja este polinômio $f(x)=x^{2}+b x+c$. Então,

$$
\begin{gathered}
f(x)^{2}=x^{4}+a x^{3}+7 x^{2}-a x+1 \\
\left(x^{2}+b x+c\right)^{2}=x^{4}+b^{2} x^{2}+c^{2}+2 b x^{3}+2 c x^{2}+2 b c x \\
=x^{4}+2 b x^{3}+\left(2 c+b^{2}\right) x^{2}+2 b c x+c^{2}
\end{gathered}
$$

Comparando a última expressão com $p(x)$, temos:

$$
c^{2}=1 \Rightarrow c= \pm 1
$$

Caso $c=1$, temos $2 c+b^{2}=7 \Rightarrow b^{2}=5 \Rightarrow b=$ $\pm \sqrt{5}$ que não serve pois $b \in \mathbb{Z}$.

Para $c=-1$ temos $-2+b^{2}=7 \Rightarrow b^{2}=9 \Rightarrow b=$ $\pm 3, \log 0$

$$
\begin{gathered}
b=3 \Rightarrow 2 b=a \Rightarrow a=6 \\
b=-3 \Rightarrow 2 b=a \Rightarrow a=-6
\end{gathered}
$$

Assim, temos que os valores possíveis de $a$ são 6 e -6 .

8. O polinômio $p(x)=x^{4}-8 x^{3}-27 x^{2}+182 x+392$ tem 4 raízes inteiras. Duas delas são positivas e iguais a um número primo que divide 84. Encontre as quatro raizes de $p(x)$.

Decompondo 84 em fatores primos temos $84=$ $2^{2} \cdot 3 \cdot 7$. Substituindo os 2,3 e 7 no polinômio observamos que:

$$
\begin{aligned}
& p(2)=2^{4}-8(2)^{3}-27\left(2 \left(^{2}+182(2)+392=600\right.\right. \\
& p(3)=3^{4}-8(3)^{3}-27(3)^{2}+182(3)+392=560 \\
& p(7)=7^{4}-8(7)^{3}-27(7)^{2}+182(7)+392=0
\end{aligned}
$$

Logo, 7 é a raiz dupla e $(x-7)^{2}$ divide $p(x)$. Desenvolvendo o produto notável e efetuando a divisão, encontramos:

$$
p(x)=\left(x^{2}-14 x+49\right)\left(x^{2}+6 x+8\right) .
$$

Analisando a fatoração do polinômio $q(x)=x^{2}+$ $6 x+8$ temos que as duas raízes são números cuja soma seja -6 e o produto 8 , logo esses números são -2 e -4 . E a raízes do polinômio são $\{-4,-2,3,3\}$.

Abaixo temos atividades direcionadas a todo o Ensino Médio, onde as relações de Girard são bastante exploradas. Além delas, trabalhamos também divisores, sistema de equações, progressões aritmética e geométrica.

9. Calcular as raízes do polinômio $p(x)=x^{3}-$ $16 x^{2}+61 x-66$ sabendo que todas são distintas e naturais.

De acordo com as relações de Girard, temos:

$$
\begin{gathered}
x_{1} x_{2} x_{3}=-\frac{-66}{1}=66 \\
x_{1}+x_{2}+x_{3}=-\frac{-16}{1}=16
\end{gathered}
$$


Podemos afirmar que as raízes são divisores de 66, assim

$$
x_{1}, x_{2}, x_{3} \in\{1,2,3,6,11,22,33,66\} .
$$

Mas os valores, 22, 33 e 66 não servem pois são maiores que 16. Além disso o 6 também não serve pois a soma dele com outros dois valores quaisquer não dá 16. A mesma observação pode ser feita para o 1 . Sendo assim, como $66=2 \cdot 3 \cdot 11$ e $2+3+11=16$, temos que as raízes são 2,3 e 11 .

10. Encontre as raízes do polinômio $p(x)=x^{3}-$ $11 x^{2}+39 x-45$, sabendo que todas são inteiras positivas e uma delas é dupla.

Pelas relações de Girard temos que:

$$
\begin{gathered}
x_{1}+x_{2}+x_{3}=-\frac{-11}{1}=11 \\
x_{1} x_{2}+x_{1} x_{3}+x_{2} x_{3}=\frac{39}{1}=39 \\
x_{1} x_{2} x_{3}=-\frac{-45}{1}=45
\end{gathered}
$$

Pela terceira relação temos que

$$
x_{1}, x_{2}, x_{3} \in\{1,3,5,9,15,45\} .
$$

Mas 15 e 45 estão descartados, visto que a soma das três raízes deve ser igual a 11. Como uma raiz é dupla, façamos $x_{1}=x_{2}$ e podemos reescrever a primeira relação da seguinte forma:

$$
2 x_{1}+x_{3}=11 \Rightarrow x_{3}=11-2 x_{1}
$$

Reescrevendo a segunda relação,

$$
x_{1}^{2}+2 x_{1} x_{3}=39 .
$$

Substituindo a equação (4) na equação (5), temos:

$$
\begin{gathered}
x_{1}^{2}+2 x_{1}\left(11-2 x_{1}\right)-39=0 \\
x_{1}^{2}+22 x_{1}-4 x_{1}^{2}-39=0 \\
3 x_{1}^{2}-22 x_{1}+39=0 .
\end{gathered}
$$

Resolvendo a equação, encontramos as raízes $\frac{13}{3}$ e 3. Mas $x_{1}$ não pode ser igual a $\frac{13}{3}$, pois este valor não é divisor de 45 . Então $x_{1}=3$.

Como $x_{1}=x_{2}$, a raiz dupla é 3 , e substituindo em $x_{3}=11-2 x_{1}$ encontramos $x_{3}=5$. Logo, as raízes procuradas são $\{3,3,5\}$.

Outra resolução: Outra opção de resolução envolve analisar a decomposição do 45 .

Como uma raiz é dupla teremos $x_{1}^{2} \cdot x_{2}=45$, mas $45=3^{2} \cdot 5$, sendo assim, as raízes são 5 e 3 ou -3 .
Para verificar se 3 ou - 3 é a raiz dupla basta efetuar a divisão de $p(x)$ por $x-5$, donde:

$$
p(x)=(x-5)\left(x^{2}-6 x+9\right),
$$

que nos leva a concluir que a raiz dupla é 3 .

11. Qual deve ser o valor de $k$ no polinômio $p(x)=$ $x^{3}-6 x^{2}+4 x+k$ para que suas raizes estejam em P.A.?

Como o polinômio é de grau 3 e as raízes devem estar em P.A., podemos escrever $x_{1}=a-r, x_{2}=a$ e $x_{3}=a+r$, e usando as relações entre os coeficientes e raízes, temos que:

$$
x_{1}+x_{2}+x_{3}=a-r+a+a+r=6 \Longrightarrow a=2
$$

Como 2 é raiz, o teste da raiz nos garante que ( $x-$ 2) divide $p(x)$ e, usando o algoritmo da divisão, temos que

$x^{3}-6 x^{2}+4 x+k=(x-2)\left(x^{2}-4 x-4\right)+(k-8)$

Como a divisão deve ser exata, devemos ter $k-$ $8=0$, donde $k=8$.

12. Encontrar as raízes do polinômio $p(x)=x^{3}-$ $9 x^{2}+23 x-15$ sabendo que suas raízes estão em

\section{P.A.}

Como as raízes estão em P.A., podemos escrever $x_{1}=a-r, x_{2}=a$ e $x_{3}=a+r$. Então, pelas relações de Girard, temos:

$$
x_{1}+x_{2}+x_{3}=9,
$$

substituindo $x_{1}, x_{2}$ e $x_{3}$,

$$
a-r+a+a+r=9 \Rightarrow 3 a=9 \Rightarrow a=3 .
$$

Logo, $x_{2}=3$ e pelo teste da raiz, $(x-3)$ divide $p(x)$. Assim, pelo algoritmo da divisão,

$$
x^{3}-9 x^{2}+23 x-15=(x-3)\left(x^{2}-6 x+5\right)
$$

O polinômio $q(x)=x^{2}-6 x+5$ tem raízes 5 e 1 (pelas relações de Girard, a soma é 6 e o produto é 5). Assim, as raízes de $p(x)$ são $\{1,3,5\}$.

13. Sabendo que o polinômio $p(x)=x^{3}-5 x^{2}+7 x-$ 3 tem 3 raízes inteiras e uma raiz dupla, encontre suas raizes.

Como a soma das três raízes deve ser 5 e o produto deve ser 3 , afirmamos que a raiz dupla deve ser 1 ou -1 e a outra raiz é 3 . Fazendo a substituição de 1 e -1 no polinômio, temos:

$$
\begin{gathered}
p(1)=1^{3}-5(1)^{2}+7(1)-3=0 \\
p(-1)=(-1)^{3}-5(-1)^{2}+7(-1)-3=-16
\end{gathered}
$$


Logo, a raiz dupla é 1.

Outra resolução: Como uma raiz é dupla, podemos considerar $x_{1}=x_{2}$ e escrever as relações:

$$
\begin{gathered}
x_{1}+x_{2}+x_{3}=5 \Longrightarrow 2 x_{1}+x_{3}=5 \Longrightarrow x_{3}=5-2 x_{1} \\
x_{1} x_{2}+x_{1} x_{3}+x_{2} x_{3}=7 \Longrightarrow x_{1}^{2}+2 x_{1} x_{3}=7 \\
x_{1} x_{2} x_{3}=3 \Longrightarrow x_{1}^{2} x_{3}=3
\end{gathered}
$$

Substituindo (6) em (7), temos:

$$
\begin{gathered}
x_{1}^{2}+2 x_{1}\left(5-2 x_{1}\right)=7 \\
3 x_{1}^{2}+10 x_{1}-7=0 .
\end{gathered}
$$

Resolvendo esta última equação, encontramos $x_{1}=$ $\frac{7}{3}$ e $x_{1}=1$. Vamos verificar qual dos valores é raiz da equação inicial. Para $x_{1}=\frac{7}{3}, p\left(\frac{7}{3}\right)=-\frac{32}{27}$, portanto $\frac{7}{3}$ não é raiz do polinômio. Para $x_{1}=1$, $p(1)=0, \operatorname{logo} 1$ é a raiz dupla. Substituindo $1 \mathrm{em}$ (6), encontramos $x_{3}=3$.

Assim, as raízes de $p(x)$ são $\{1,1,3\}$.

14. Escreva a forma fatorada do polinômio $P(x)=$ $x^{3}-3 x^{2}-6 x+8$ sabendo que as raízes são todas inteiras e a soma de duas de suas raízes é igual a 5 .

Esta é a questão 3 que já foi resolvida. Queremos aqui dar uma outra resolução com base nas relações entre os coeficientes e raízes do polinômio. Suponhamos $x_{1}+x_{2}=5$ e substituindo na relação $x_{1}+x_{2}+x_{3}=3$, temos:

$$
5+x_{3}=3 \Longrightarrow x_{3}=-2
$$

Como o produto das raízes é -8 , temos que $x_{1}$ e $x_{2}$ são divisores de $-8, \log x_{1}, x_{2} \in\{ \pm 1, \pm 2, \pm 4, \pm 8\}$ e $x_{1}+x_{2}=5$, o que nos leva a concluir que $x_{1}=$ 1 e $x_{2}=4$. Assim, as raízes do polinômio são $\{-2,1,4\}$.

15. As raízes do polinômio $p(x)=x^{3}-6 x^{2}+k x+64$ estão em P.G. Nessas condições, calcule o coeficiente $k$.

Como as raízes estão em P.G., podemos escrever $x_{1}=\frac{a}{q}, x_{2}=a$ e $x_{3}=a q$.

Das relações de Girard, temos:

$$
\begin{gathered}
x_{1} x_{2} x_{3}=-64 \\
\frac{a}{q} \cdot a \cdot a q=-64 \\
a^{3}=-64 \Longrightarrow a=-4 .
\end{gathered}
$$

Substituindo -4 no polinômio, encontramos:

$$
\begin{gathered}
(-4)^{3}-6(-4)^{2}+k(-4)+64=0 \\
-64-96-4 k+64=0 \\
4 k=-96 \Longrightarrow k=-24
\end{gathered}
$$

16. Dona Leide foi ao shopping comprar presente do dia das crianças para seus netos menores. $O$ número de presentes que ela vai comprar equivale ao número de raízes distintas do polinômio $p(x)=x^{4}-9 x^{3}+30 x^{2}-44 x+24$. Descubra o número de netos pequenos que dona Leide tem, sabendo que todas as raízes de $p(x)$ são inteiras.

As possíveis raízes racionais do polinômio $p(x)$ pertencem ao conjunto dos divisores de 24:

$$
D(24)=\{ \pm 1, \pm 2, \pm 3, \pm 4, \pm 6, \pm 8, \pm 12, \pm 24\} .
$$

Por substituição, $p(1)=2, p(-1)=108, p(2)=0$. Logo, 2 é raiz de $p(x)$ e, pelo algoritmo da divisão,

$$
p(x)=(x-2)\left(x^{3}-7 x^{2}+16 x-12\right) .
$$

Encontremos, agora, as raízes do polinômio $q(x)=$ $x^{3}-7 x^{2}+16 x-12$. As possíveis raízes pertencem ao conjuntos dos divisores de 12 :

$$
D(12)=\{ \pm 1, \pm 2 \pm 3, \pm 4, \pm 6, \pm 12\} .
$$

Novamente por substituição: $q(1)=-2, q(-1)=$ $-36, q(2)=0$. Logo, 2 é raiz de $x^{3}-7 x^{2}+16 x-$ 12 e, pelo algoritmo da divisão, temos:

$$
x^{3}-7 x^{2}+16 x-12=(x-2)\left(x^{2}-5 x+6\right) .
$$

As raízes de $x^{2}-5 x+6$ são $\{2,3\}$. Assim, as raízes distintas de $p(x)$ são 2 e 3 . Logo, dona Leide tem 2 netos pequenos.

17. O polinômio $p(x)=x^{4}+x^{3}-7 x^{2}-x+6$ tem quatro raízes inteiras, sendo duas simétricas. Quais são essas raízes?

Sabemos que

$$
x_{1}+x_{2}+x_{3}+x_{4}=-\frac{1}{1}=-1
$$

Podemos escrever $x_{2}=-x_{1}$. Substituindo na expressão acima,

$$
x_{1}-x_{1}+x_{3}+x_{4}=-1 \Longrightarrow x_{3}+x_{4}=-1
$$

Como $x_{1}, x_{2}, x_{3}, x_{4} \in\{-1,1,-2,2,-3,3,-6,6\}$, temos quatro opções: $\{-1,1,2,-3\} ;\{-3,3,-2,1\}$; $\{-6,6,-3,2\}$ ou $\{-6,6,-2,1\}$.

Porém, $x_{1} x_{2} x_{3} x_{4}=6$, logo apenas uma das opções satisfaz esta condição: $\{-1,1,2,-3\}$. 
18. Uma das raízes do polinômio $p(x)=x^{3}+11 x^{2}-$ $160 x+400$, que tem três raízes distintas, é -20 . Sem usar a divisão de polinômios, encontre as outras duas raízes sabendo que estas são inteiras.

Decompondo 400 em fatores primos temos: $400=$ $2^{4} \cdot 5^{2}$. Se uma raiz é -20 , por Girard as outras são divisores de 20 , pois o produto das 3 raízes é igual a -400 então só podem ser $(1,20),(-1,-20),(2,10)$, $(-2,-10),(4,5),(-4,-5)$. Dessa forma, é só testar se $1,-1,2,-2,4,-4$ são raízes. Obtendo que, dessa lista, apenas o par $(4,5)$ contém raizes do polinômio.

19. A diferença entre duas das raízes do polinômio $P(x)=x^{3}-4 x^{2}-17 x+60$ é 2. Quais são as raízes desse polinômio sabendo que todas são inteiras?

Com a diferença entre duas das raízes é 2, podemos escrever

$$
x_{1}-x_{2}=2 \Longrightarrow x_{1}=2+x_{2}
$$

Pelas relações de Girard $x_{1}+x_{2}+x_{3}=4$, então

$$
2+x_{2}+x_{2}+x_{3}=4 \Longrightarrow x_{3}=2-2 x_{2}
$$

Além disso, temos que

$$
x_{1} x_{2}+x_{1} x_{3}+x_{2} x_{3}=-17
$$

Substituindo as equações (9) e (10) em (11), temos:

$$
\begin{gathered}
\left(2+x_{2}\right) x_{2}+\left(2+x_{2}\right)\left(2-2 x_{2}\right)+x_{2}\left(2-2 x_{2}\right)=-17 \\
2 x_{2}+x_{2}^{2}+4-4 x_{2}+2 x_{2}-2 x_{2}^{2}+2 x_{2}-2 x_{2}^{2}=-17 \\
-3 x_{2}^{2}+2 x_{2}+21=0
\end{gathered}
$$

Resolvendo a equação do segundo grau, encontra$\operatorname{mos} x_{2}=-\frac{7}{3}$ e $x_{2}=3$. Mas $-\frac{7}{3}$ não pode ser raiz, $\log \mathrm{o}, x_{2}=3$.

Substituindo o valor de $x_{2}$ em (9) e(10), encontra$\operatorname{mos} x_{1}=5$ e $x_{3}=-4$.

Outra resolução: Analisando a decomposição em fatores primos de 60 , temos $60=2^{2} \cdot 3 \cdot 5$. Como a soma das três raízes deve ser 4 e o produto -60 , devemos ter 1 ou 3 raízes negativas. Mas se as três forem negativas, não poderemos ter soma 4 , portanto teremos apenas uma raiz negativa e duas positivas.

Dentre os divisores de 60, tomando um divisor negativo e fazendo a combinação com outros dois positivos de forma que a soma dos três seja 4 , chegamos às raízes que só podem ser $\{-4,3,5\}$.
20. O polinômio $p(x)=x^{5}-7 x^{4}+10^{3}+18 x^{2}-27 x-$ 27 tem raízes inteiras, sendo uma dupla e uma tripla. Encontrar suas raízes.

Pelas relações de Girard temos que a soma das raízes é 7 e o produto entre elas é 27. Observando a decomposição em fatores primos de $27=3^{3}$, temos que as raízes só podem ser \pm 1 a dupla e \pm 3 a tripla.

Para avaliarmos o sinal da raiz vamos substituir 1 e -1 no polinômio.

$$
\begin{aligned}
& p(1)=1-7+10+18-27-27=-32 \\
& p(-1)=-1-7-10+18+27-27=0
\end{aligned}
$$

Logo, -1 é a raiz dupla de $p(x)$ e como o produto é positivo, -3 não pode ser a raiz tripla, assim temos que a raiz tripla é 3 .

21. As raízes do polinômio $p(x)=x^{3}-14 x^{2}+64 x-$ 96 são inteiras e positivas. Sabendo que o polinômio tem raiz dupla e que o $\mathrm{mmc}$ entre as raízes é igual a 12 e o mdc é igual a 2, determine-as.

Observemos a decomposição em fatores primos do número $96=2^{5} \cdot 3$.

Como o mdc é 2, o número 3 não pode ser raiz, e como o expoente do 3 na decomposição é 1, ele não pode ser fator da raiz dupla, logo temos as seguintes opções de raízes: $(1,1,96) ;(2,2,24)$ ou $(4,4,6)$.

Como o mmc é 12, a opção que nos dá as raízes do polinômio é $(4,4,6)$.

22. O polinômio $p(x)=x^{5}-26 x^{4}+252 x^{3}-1114 x^{2}+$ $2147 x-1260$ tem cinco raízes inteiras positivas distintas. Encontre estas raízes sabendo que são duas a duas primas entre si.

Decompondo 1260 em fatores primos, encontra$\operatorname{mos} 1260=2^{2} \cdot 3^{2} \cdot 5 \cdot 7$.

Como as raízes são todas duas a duas primas entre si, a raiz que tiver um fator primo $p$ deve conter todos os fatores de $p$. Também não podemos ter números com mais de um fator primo como 6, 15, 20, 35 ou 45 como raiz. Sendo assim, as possíveis raízes são 4,9,5,7 e 1. Como as raízes são todas positivas e a soma deve ser 26 , temos $1+4+5+7+9=26$. Assim as raízes do polinômio são $(1,4,5,7,9)$.

23. O polinômio $p(x)=x^{4}+15 x^{3}+80 x^{2}+180 x+$ 144 tem quatro raízes inteiras negativas. Sabendo que o mmc entre duas delas é 12 e que o mdc entre as mesmas duas raízes é 2 , encontre todas as raízes deste polinômio.

Escrevamos $a$ e $b$ as duas raízes cujos valores do mmc e mdc são dados. Como $\operatorname{mmc}(a, b) \cdot \operatorname{mdc}(a, b)=$ 
$a \cdot b$, então $a \cdot b=24$. Como o produto das quatro raízes deve ser igual a 144 , temos que o produto das duas raízes restantes será 144:24=6, e assim temos as seguintes opções: $(-1,-6)$ ou $(-2,-3)$, já que são todas negativas. Testando -1 e -2 verificamos que: $p(-1)=30$ e $p(-2)=0$. Logo, duas raízes deste polinômio são -2 e -3 .

Podemos, agora, efetuar a divisão de $p(x)$ por $(x+2)(x+3)=x^{2}+5 x+6$ e encontrar as raízes do quociente usando a fórmula de Bhaskara, ou ainda analisar que, como o produto de $a$ e $b$ é igual a 24 , temos as opções $(-2,-12)$ ou $(-4,-6)$. Como a soma das quatro raízes deve ser -15 e já concluímos que -2 e -3 são raízes, temos que: $-2-$ $3-2-12=-19$ e $-2-3-4-6=-15$.

Assim, as raízes de $p(x)$ são $(-2,-3,-4,-6)$.

24. O polinômio $p(x)=x^{4}-28 x^{3}+260 x^{2}-89 x+$ 960 tem quatro raízes inteiras positivas e distintas. Sabendo que o mmc entre elas é 60 e o mdc é 2 , encontre as raízes.

Como o mdc entre as raízes é 2 , temos que todas são números pares. Pela decomposição do número $960=2^{6} \cdot 3 \cdot 5$ observamos que os 6 fatores 2 estão assim distribuídos: três raízes com um fator 2 e uma com três fatores 2 ou duas raízes com um fator 2 e duas com dois fatores 2 .

Resta-nos distribuir os fatores 3 e 5 . Para a primeira opção de distriuição do $2^{6}$ só podemos ter as raizes $(2,6,8,10)$, já que todas devem ser distintas. Para a segunda distribuição podemos ter $(2,4,6,20)$ ou $(2,4,10,12)$.

Para decidirmos qual das três combinações forma as raízes do polinômio, observemos que a soma destas deve ser igual a 28, assim:

$$
\begin{aligned}
& 2+6+8+10=26 \\
& 2+4+6+20=32 \\
& 2+4+10+12=28
\end{aligned}
$$

implica as raízes de $p(x)$ serem $(2,4,10,12)$.

Mostraremos agora alguns exemplos de atividades envolvendo raízes racionais, onde, além das relações de Girard, utilizamos também a seguinte proposição:

Proposição 5.1. Sejam $n>1$ inteiro, $f(x)=a_{n} x^{n}+$ $\ldots+a_{1} x+a_{0}$ um polinômio de coeficientes inteiros $e$ p e q inteiros não nulos primos entre si. Se $f\left(\frac{p}{q}\right)=0$, então:

(a) $p \mid a_{0}$ e $q \mid a_{n}$. (b) Se $f$ for mônico, então as possíveis raízes racionais de $f$ são inteiras.

que geralmente é trabalhada no ensino médio logo após o estudo de relações entre coeficientes e raízes.

25. As dimensões $a, b$ e $c$, em $\mathrm{cm}$, de um paralelepípedo retângulo, são as raízes do polinômio $p(x)=6 x^{3}-44 x^{2}+103 x-77$.

(a) Calcule o volume do paralelepípedo.

(b) Calcule a soma das áreas das faces desse paralelepípedo.

Pelas relações de Girard,

$$
\begin{gathered}
a+b+c=-\frac{-44}{6}=\frac{22}{3} \\
a b+a c+b c=\frac{103}{6} \\
a b c=-\frac{-77}{6}=\frac{77}{6}
\end{gathered}
$$

a) $V=a b c=\frac{77}{6} \mathrm{~cm}^{3}$

b) $A_{\text {total }}=2(a b+a c+b c)=2 \cdot \frac{103}{6}=\frac{103}{3} \mathrm{~cm}^{2}$

26. Pesquisar as raízes dos polinômios, sabendo que são todas distintas e racionais.

(a) $p(x)=2 x^{3}-x^{2}-2 x+1$.

De acordo com a proposição 5.1, temos que as raízes pertencem ao conjunto $\left\{-1,1,-\frac{1}{2}, \frac{1}{2}\right\}$. Pelas relações de Girard,

$$
\begin{gathered}
x_{1}+x_{2}+x_{3}=-\frac{1}{2} \\
x_{1} x_{2} x_{3}=\frac{1}{2}
\end{gathered}
$$

Como temos 3 raízes distintas cuja soma é $-\frac{1}{2}$ e o produto é $\frac{1}{2}$, a única solução possível é $S=\left\{-1,1,-\frac{1}{2}\right\}$.

(b) $g(x)=2 x^{3}-7 x^{2}+7 x-2$

O conjunto solução $S$ pertence ao conjunto $\left\{-\frac{1}{2}, \frac{1}{2}-1,1,-2,2\right\}$ de acordo com a proposição 5.1, e, segundo as relações de Girard,

$$
\begin{aligned}
& x_{1}+x_{2}+x_{3}=\frac{7}{2} \\
& x_{1} x_{2} x_{3}=\frac{2}{2}=1 .
\end{aligned}
$$

Como o produto das três raízes deve ser 1 , temos as opções: $\left\{-1,-\frac{1}{2}, 2\right\} ;\left\{-1, \frac{1}{2},-2\right\}$; 
$\left\{1, \frac{1}{2}, 2\right\}$ ou $\left\{-\frac{1}{2}, 1,-2\right\}$. Como a soma das raízes deve ser $\frac{7}{2}$, calculemos a soma das opções apresentadas:

$$
\begin{gathered}
-1-\frac{1}{2}+2=\frac{1}{2} \\
-1+\frac{1}{2}-2=-\frac{5}{2} \\
1+\frac{1}{2}+2=\frac{7}{2} \\
1-\frac{1}{2}-2=-\frac{3}{2}
\end{gathered}
$$

Observando as somas, concluímos que as raízes de $g(x)$ são $\left\{\frac{1}{2}, 1,2\right\}$.

c) $h(x)=2 x^{3}+3 x^{2}-8 x+3$

Sabemos que a solução pertence ao conjunto

$$
\left\{-\frac{1}{3}, \frac{1}{3},-\frac{1}{2}, \frac{1}{2},-1,1,-3,3\right\} \text {. }
$$

Além disso, temos as relações:

$$
\begin{gathered}
x_{1}+x_{2}+x_{3}=-\frac{3}{2} \\
x_{1} x_{2} x_{3}=-\frac{3}{2}
\end{gathered}
$$

Assim, temos as seguintes possibilidades:

$$
\left\{-3, \frac{1}{2}, 1\right\} \quad\left\{-\frac{1}{2}, 1,3\right\} \quad \text { ou } \quad\left\{-1, \frac{1}{2}, 3\right\} \text {. }
$$

Analisemos as somas:

$$
\begin{gathered}
-3+\frac{1}{2}+1=-\frac{3}{2} \\
-\frac{1}{2}+1+3=\frac{7}{2}
\end{gathered}
$$

Pela soma, as raízes do polinômio $h(x)$ são $\left\{-3, \frac{1}{2}, 1\right\}$. Verifiquemos o produto:

$$
-3 \cdot \frac{1}{2} \cdot 1=-\frac{3}{2}
$$

Obs.: É possível criar inúmeros problemas para explorar e estimular a habilidade dos alunos a partir dos modelos expostos no trabalho. Para tanto, basta um pouco de imaginação por parte do professor.

\section{Conclusões}

A partir dessa investigação, concluímos que há uma imensa variedade de atividades que podem ser criadas com o intuito de integrar conteúdos de polinômios e aritmética. Isso possibilita aos alunos deixarem de ver os diferentes conteúdos como tópicos isolados. Além disso, vimos que, nesse tipo de exercícios, há várias possibilidades de resolução e acreditamos que isso pode incentivar os alunos a buscarem soluções mais rápidas ou menos trabalhosas, estimulando a criatividade e raciocínio lógico.

\section{Referências}

[1] BOYER, Carl Benjamin. História da Matemática. $2^{\mathrm{a}}$ ed. Trad. ELZA F. OMIDE. São Paulo: Edgard Blücher, 1996

[2] DOMINGUES, Hygino H. Fundamentos de Aritmética. São Paulo: Atual. 1991.

[3] DANTE, Luis Roberto. Matemática: Contexto e Aplicações. São Paulo: Ática. 2011.

[4] DOMINGUES, Hygino H. e IEZZI, Gelson. Álgebra Moderna. $3^{\mathrm{a}}$ ed. São Paulo: Atual editora, 1982.

[5] EVES, Howard Whitley. Introdução à história da Matemática. $5^{\mathrm{a}}$ ed.Campinas: Unicamp, 1997.

[6] GARBI, Gilberto G. O Romance das Equações Algébricas. São Paulo: Makron Books, 1997.

[7] GIOVANNI, José Ruy e BONJORNO, José Roberto. Matemática: uma nova abordagem: vol3. São Paulo: FTD, 2001.

[8] GONÇALVES, Adilson. Introdução à Álgebra. 5a ed. Rio de Janeiro: Impa, Projeto Euclides, 2008.

[9] HEFEZ, Abramo e VILLELA, Maria Lúcia Torres. Polinômios e Equações Algebricas. $1^{\mathrm{a}}$ ed. Rio de Janeiro: SBM, 2012. (Coleção Profmat)

[10] MARTINEZ, FABIO Brochero; et al. Teoria dos Números: um passeio com primos e outros números familiares pelo mundo inteiro. $3^{\mathrm{a}}$ ed. Rio de Janeiro: IMPA, 2013.

[11] NETO, Antonio Caminha Muniz. Tópicos de Matemática Elementar: Volume 6 - Polinômios. $1^{\mathrm{a}}$ ed. Rio de Janeiro: SBM, 2012. (Coleção Professor de Matemática)

[12] ROQUE, Tatiana. História da Matemática: Uma Visão Crítica, Desfazendo Mitos e Lendas. Rio de janeiro: Zahar, 2012.

[13] SANTOS, José Plinio de Oliveira. Introdução à Teoria dos Números. Rio de Janeiro: Impa, 1998. (Coleção Matemática Universitária)

[14] SANTOS, Rosipléia Souza dos. Relações de Girard e Atitmética: atividades para o ensino fundamental e médio. 2014. 65 p. Dissertação - Mestrado Profissional em Matemática. Universidade Estadual de Feira de Santana, Feira de Santana, 2014. 
[15] Relações de Girard. Disponível em http://www.colegioweb.com.br/trabalhosescolares/matematica/equacoes-

algebricas/relacoes-de-girard.html.) Acesso em 19 dez. 2013. 